\title{
A Control Algorithm for Multiscale Simulations of Liquid Water
}

\author{
Evangelos M. Kotsalis and Petros Koumoutsakos \\ Computational Science, ETH Zürich, CH-8092, Switzerland \\ petros@ethz.ch
}

\begin{abstract}
We present multiscale simulations of liquid water using a novel control algorithm to couple non-periodic molecular dynamics (MD) and continuum descriptions in the context of a Schwarz alternating method. In the present multiscale approach the non-periodic MD simulations are enhanced by an effective external boundary force that accounts for the virial component of the pressure and eliminates spurious density oscillations close to the boundary. This force is determined by a simple control algorithm that enables coupling of the atomistic description to a coarse grained or a continuum description of liquid water. The proposed computational method is validated in the case of equilibrium and parallel flow.
\end{abstract}

\section{Introduction}

Multiscale modeling is critical for computational studies of new technologies such as those at the interface of nanotechnology and biology. Nanodevices present us the opportunity to interact with biological systems at the molecular level for applications such as molecular drug delivery and artificial nanosyringes. These nanodevices are in turn often embedded in continuous systems (e.g. when nanofluidic channels are interfacing microfluidic domains) and their simulation dictates an inherently multiscale problem.

Multiscale approaches coupling MD simulations of atomistic descriptions with grid/particle discretizations of the continuum domain have been mainly developed for Lennard Jones fluids. These approaches can be distinguished in the way information is exchanged between the two descriptions, resulting in two broad classes of coupling schemes: the method of direct flux exchange [1234 and the Schwarz alternating method [5678]. In recent years a number of efforts have addressed multiscale simulations to systems involving polyatomic, polar molecules such as water. In these simulations the handling of electrostatic and steric effects becomes critical and requires several extensions over multiscale modeling of monatomic systems. Fabritiis et al. 4] applied the flux exchange scheme to simulate liquid water. Praprotnik et al. 9] presented multiscale simulations of liquid water using a spatially adaptive molecular resolution procedure to change from a coarse-grained to an all-atom representation on-the-fly.

In this paper we extend the Schwarz alternating method implemented in $7 / 8$ to conduct multiscale simulations of liquid water. The key challenge of these

M. Bubak et al. (Eds.): ICCS 2008, Part II, LNCS 5102, pp. 234 241 2008.

(C) Springer-Verlag Berlin Heidelberg 2008 
simulations is the appearance of spurious density fluctuations due to the presence of non-periodic boundary conditions in the MD domain. The present method circumvents this difficulty by introducing a boundary force that is determined via a control algorithm. We note that this approach does not involve the calculation of the pressure tensor that is required in the flux base methods. This helps reduce the computational cost of the method, as the calculation of the pressure tensor 5] requires a large amount of samples or the use of large cell sizes at the expense of spatial resolution. In addition, in the case of the flux-based methods a buffer is necessary that should be large enough, such that the atoms and molecules inside the bulk liquid do not interact with the rarefied region [8]. In the present method the "buffer" is restricted to the region where we impose the velocity boundary condition from the continuum.

\section{Coupling Continuum Liquids with Molecular Dynamics of Water}

The coupling of continuum and atomistic descriptions of liquid water , implies MD simulations subject to non-periodic boundary conditions (BC). In MD simulations the position $\mathbf{r}_{i}=\left(x_{i}, y_{i}, z_{i}\right)$ and velocities $\mathbf{v}_{i}=\left(u_{i}, v_{i}, w_{i}\right)$ of the $i$-th particle evolve according to Newton's equation of motion:

$$
\begin{aligned}
\frac{d}{d t} \mathbf{r}_{i} & =\mathbf{v}_{i}(t) \\
m_{i} \frac{d}{d t} \mathbf{v}_{i} & =\mathbf{F}_{i}=-\sum_{j \neq i} \nabla U\left(r_{i j}\right),
\end{aligned}
$$

where $m_{i}$ is the mass and $\mathbf{F}_{i}$ the force on particle $i$. The interaction potential $U\left(r_{i j}\right)$ models the physics of the system. Here we consider MD simulations using the rigid SPC/E water model et al. 10, with an $\mathrm{O}-\mathrm{H}$ bond length of $1 \AA$ and a H-O-H angle of $109.47^{\circ}$ constrained using the SHAKE algorithm 11. The $\mathrm{SPC} / \mathrm{E}$ model involves a Lennard-Jones potential between the oxygen atoms

$$
U_{\alpha \beta}\left(r_{i j}\right)=4 \epsilon_{\alpha \beta}\left[\left(\frac{\sigma_{\alpha \beta}}{r_{i j}}\right)^{12}-\left(\frac{\sigma_{\alpha \beta}}{r_{i j}}\right)^{6}\right], \text { for } r_{i j}<r_{c},
$$

where $r_{c}$ is the radius of truncation $\left(U_{\alpha \beta}\left(r_{i j}\right)=0\right.$ for $\left.r_{i j}>r_{c}\right)$, and $\alpha$ and $\beta$ refer to the atomic species (here oxygen-oxygen: $\epsilon_{\mathrm{OO}}=0.6501 \mathrm{~kJ} \mathrm{~mol}^{-1}$ and $\left.\sigma_{\mathrm{OO}}=0.3166 \mathrm{~nm}\right)$. The model involves a Coulomb potential acting between all atom pairs of different water molecules

$$
U\left(r_{i j}\right)=\frac{q_{i} q_{j}}{4 \pi \epsilon_{0}} \frac{1}{r_{i j}},
$$

where $\epsilon_{0}$ is the permittivity in vacuum, and $q_{i}$ is the partial free charge, $q_{O}=$ -0.8476 and $q_{H}=0.4238$, respectively. These partial free charges describe the fixed, static dipol moment of water. 10. In this work all interaction potentials are 
truncated for distances beyond a cutoff radius $\left(r_{c}\right)$ of $1.0 \mathrm{~nm}$ and the equations of motion are integrated using a Verlet algorithm with a timestep $d t$ of $2 \mathrm{fs}$.

The elimination of periodic boundary conditions in the MD simulations results in a non-uniform density across the domain and hinders the calculation of the virial pressure. In order to correct the calculation of the mean virial pressure, we impose a boundary force $F_{m}$, along with a specular wall that prevents molecules from leaving the atomistic domain and maintains the kinetic component of the system pressure. We detect the collision of a water molecule by checking whether its center of mass has crossed the boundary. Molecules that hit a moving wall during time step $n$ are identified by computing the collision time $t^{\prime}=\left(\mathrm{com}^{n}-\right.$ $\left.x_{w}\right) /\left(u_{w}-\tilde{u}_{x}^{n+1 / 2}\right)$, where $\operatorname{com}^{n}$ is the center of mass of the water molecule, $x_{w}$ and $u_{w}$ the initial wall position and wall speed, and $\tilde{u}_{x}^{n+1 / 2}$ is the center of mass velocity of the molecule after the regular leap-frog update but before a possible reflection. If $t^{\prime}$ is smaller than the time step $d t$ then the molecule is crossing the boundary and the new velocity and position of each atom are calculated as:

$$
\begin{aligned}
& u^{n+1 / 2}=\tilde{u}^{n+1 / 2}-2\left(u_{x}^{n+1 / 2}-u_{w}\right) \\
& x^{n+1}=x^{n}+t^{\prime} \tilde{u}^{n+1 / 2}+\left(d t-t^{\prime}\right) u^{n+1 / 2}
\end{aligned}
$$

The wall force $\left(F_{m}\right)$ can be computed from the pair potential and the pair correlation function $g(r)$ of the working fluid [7]. This technique was shown to alleviate many of the drawbacks of existing methods but it is not sufficient for all state points of dense liquids. In [8] a simple control algorithm was proposed that can achieve the target density in the system for a wide range of temperatures. This algorithm, originally developed for monoatomic liquids, is extended here for the case of water. Since the kinetic energy in the system is conserved, we enforce right virial pressure in the non-periodic system, by applying an external boundary force to eliminate all the disturbances in the density.

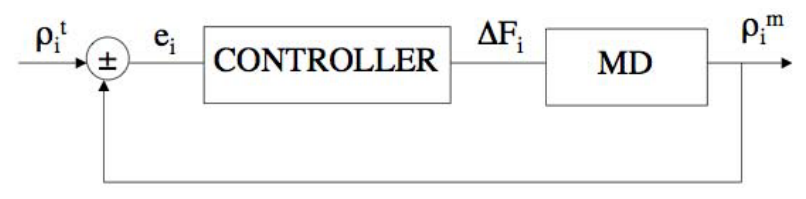

Fig. 1. Schematic of the control algorithm for reducing density fluctuations

\section{Control Algorithm and Validation}

We examine the validity of this method in a water system at equilibrium in the liquid regime $\left(T=300 \mathrm{~K}, \rho=997 \mathrm{~kg} \mathrm{~m}^{-3}\right)$. The size of the computational domain is $3 \mathrm{~nm} \times 3 \mathrm{~nm} \times 3 \mathrm{~nm}$ and periodicity is removed in the $x$-direction. The system is weakly coupled to a Berendsen thermostat 12 with a time constant of 0.1 ps. The heat bath is imposed cellwise using $6 \times 1 \times 1$ cells and after equilibration we heat only the atoms located in the cells close to the $x$ boundary. In order 
to reduce spurious oscillations in the density we apply the control algorithm to the mean external boundary force applied to the MD system.

The control approach is sketched in Fig. 1. Each iteration involves the following steps: we start by applying no external boundary force. Then we measure the
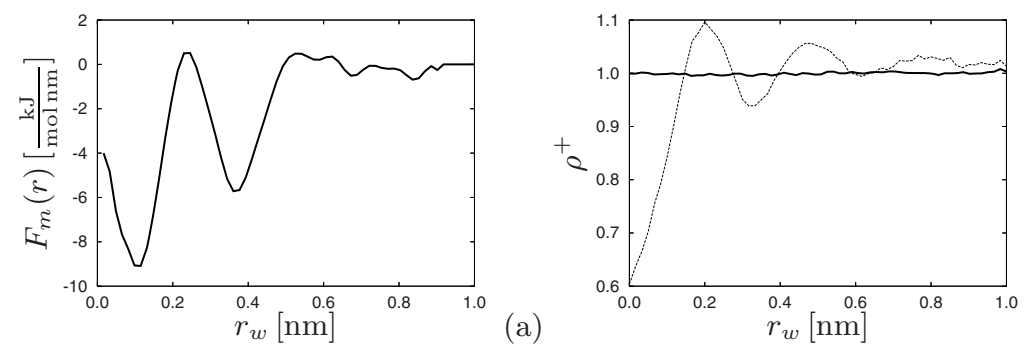

Fig. 2. a) The resulting external boundary force computed after applying a control algorithm. (b) The corresponding controlled (-) and uncontrolled (no external boundary force) reduced density values $\left(\rho^{+}=\rho / \rho_{\text {bulk }}\right)(---)$. The value used for $K_{P}$ is $0.00332 \frac{\mathrm{nm}^{3} \mathrm{~mol}}{\mathrm{amu} \mathrm{kJ}}$ and both the force and density have been sampled over $2.4 \mathrm{~ns}$.

density in short time intervals filtering away high frequency noise. This filtering makes the method more suitable for coupling the atomistic to the continuum description since it is found to improve the convergence of the method. The density $\rho^{m^{\prime}}$ is measured with a spatial resolution $\delta x$ of $0.0166 \mathrm{~nm}$ in time intervals of $6 \mathrm{ps}$ and processed twice through a gaussian filter resulting in:

$$
\begin{aligned}
& \rho^{m^{\prime \prime}}(x)=\frac{1}{\epsilon} \int \rho^{m^{\prime}}(y) \exp \left(-\frac{(x-y)^{2}}{\epsilon^{2}}\right) d y \\
& \rho^{m}(x)=\frac{1}{\epsilon} \int \rho^{m^{\prime \prime}}(y) \exp \left(-\frac{(x-y)^{2}}{\epsilon^{2}}\right) d y
\end{aligned}
$$

where $\epsilon=2 \delta x$. The cutoff used for the discrete evaluation of the convolution is $3 \delta x$ 8. We then evaluate the error as:

$$
e\left(r_{w}\right)=\rho^{t}-\rho^{m}\left(r_{w}\right)
$$

where $r_{w}$ is the distance to the boundary, $\rho^{t}$ the desired constant target density and $\rho^{m}$ the measured filtered value. We compute the gradient of this error as $\epsilon\left(r_{w}\right)=\nabla e\left(r_{w}\right)=-\nabla \rho^{m}\left(r_{w}\right)$ and amplify it with a factor $K_{P}$ to obtain the adjustment $\Delta F$ to the boundary force as:

$$
\Delta F_{i}=K_{P} \epsilon_{i},
$$

for each $i$ th bin with $\left|x_{i}-x_{w}\right|<0.9 r_{c}$. The boundary force is finally computed as:

$$
F_{i}^{\text {new }}=F_{i}^{\text {old }}+\Delta F_{i}
$$




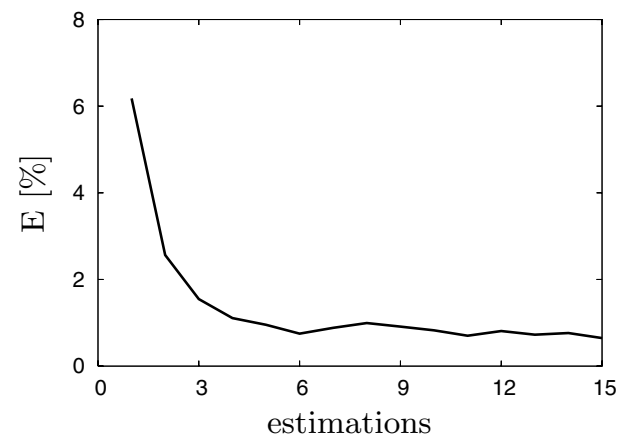

Fig. 3. Convergence of the root mean square of the error $\left(E=\sqrt{\frac{1}{N} \sum_{i=1}^{N} e_{i}^{2}}\right)$ in density when adjusting the external boundary force $\left(K_{P}=0.00332 \frac{\mathrm{nm}^{3} \mathrm{~mol}}{\mathrm{amu} \mathrm{kJ}}\right)$. Each estimation of $E$ corresponds to 30 updates of the online control force.

and applied to the center of mass of each water molecule. Here we note that through all the iterations $\Delta F_{i}$ is constrained to the trivial value if $\left|x_{i}-x_{w}\right|>$ $0.9 r_{c}$ to avoid any undesirable influence of noise in this region close to the cutoff where no oscillation in the density is observed (see Fig.2). We consider that the method has converged once the root mean square of the error $E=\sqrt{\frac{1}{N} \sum_{i=1}^{N} e_{i}^{2}}$ is less than a prescribed value, here $1 \%$. The online controller keeps acting on the system and $E$ is computed in time intervals of 180 ps. We test this approach for the liquid state. We start by applying no external force, and density oscillations close to the boundary are observed. We set $K_{P}=0.00332 \frac{\mathrm{nm}^{3} \mathrm{~mol}}{\mathrm{amu} \mathrm{kJ}}$ and the results shown in Fig. 2 demonstrate that the control approach eliminates the density oscillations. Here we note that the averaged value (over $2.4 \mathrm{~ns}$ ) of the integral of the external boundary force $\rho_{n} \int_{0}^{r_{c}} F_{m}(r) d r$ is equal to the virial pressure of liquid water in an atomistic simulation subject to periodic boundary conditions, where $\rho_{n}$ is the number density of water. In [8] we showed that the value of $K_{P}$ determines the stablity properties and the convergence rate of the algorithm. In Fig. 3 we show the convergence of the method for the value of $K_{P}=0.00332 \frac{\mathrm{nm}^{3} \mathrm{~mol}}{\mathrm{amukJ}}$. In this case the method has converged approximately after $900 \mathrm{ps}$. As a final diagnostic we measure the angle $\phi$ between the normal of the wall and the dipole moment of each water molecule. In Fig. 4 we show the probability distribution of $\cos (\phi)$ at the wall, at the cutoff distance from the boundary and at the center of the computational domain $(1.5 \mathrm{~nm}$ from the wall). The NPBC result into a spurious preference in the orientation of the water molecules at the wall that vanishes as the distance from the boundary increases. The mean deviation from the periodic reference case is $1.0^{\circ}$ at a cutoff distance. At the center of the domain the distribution is uniform with no deviation from the reference case. In addition, we examine the performance of the control algorithm in the case of parallel flow at the same liquid state. The periodicity is broken in the flow 


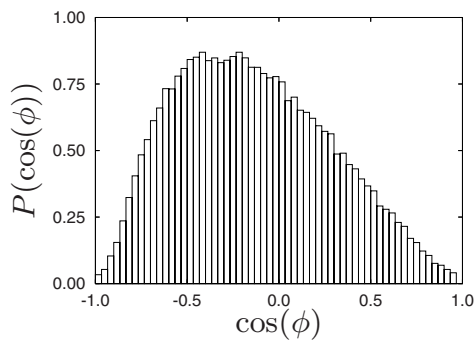

(a)

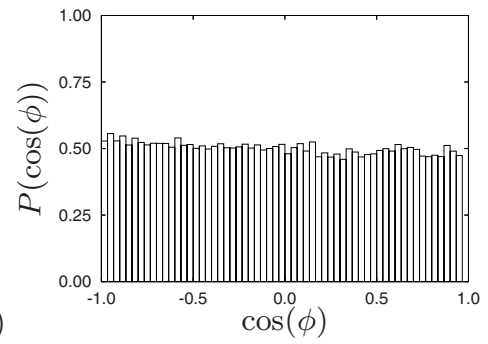

(b)

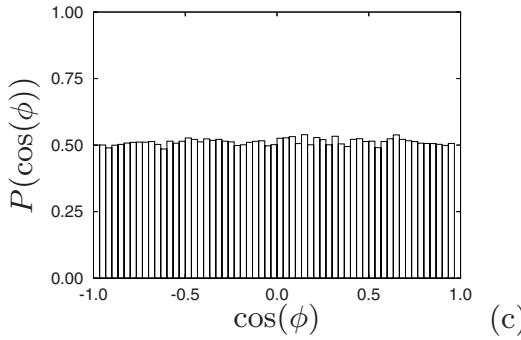

Fig. 4. The probability distribution of the cosine of the angle between the dipole of each water molecules with the normal to the $x$-boundary at the wall (a), at the cutoff distance of $1 \mathrm{~nm}$ (b) and at the center of the domain (c). As the distance from the wall increases the liquid recovers the correct uniform distribution.

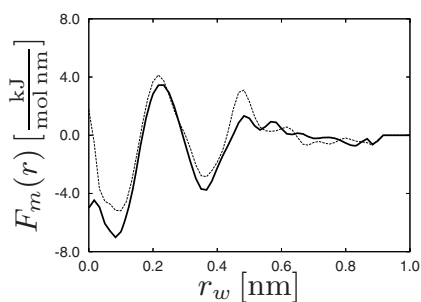

(a)
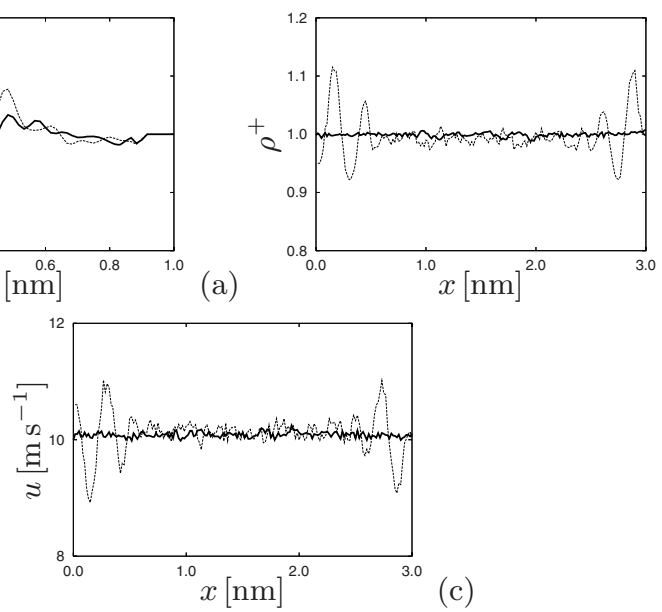

(c)

Fig. 5. (a) The resulting external boundary forces in the case of parallel flow: (---) inflow, (-) outflow. (b) The resulting reduced density $\left(\rho^{+}=\rho / \rho_{\text {bulk }}\right)$ profiles in the $\mathrm{x}$ direction: (solid line) controlled case, (dashed line) uncontrolled case (no external boundary force). (c) Resulting velocity profiles in the $x$-direction in the case of parallel flow: (-) controlled case, (---) uncontrolled case (no external boundary force). The value used for $K_{P}$ is $0.0166 \frac{\mathrm{nm}^{3} \mathrm{~mol}}{\mathrm{amu} \mathrm{kJ}}$ and the force, density and velocity have been sampled over $2.4 \mathrm{~ns}$. 
direction $(x)$. The system is weakly coupled to a Berendsen thermostat [12] with a time constant of $0.1 \mathrm{ps}$. The heat bath is imposed cellwise using $6 \times 1 \times 1$ cells. The flow is imposed by adjusting the mean flow velocity of the atoms 13 in the computational cells with center points $(x=0.25)$ and $(x=2.75)$ to achieve a mean velocity of $10 \mathrm{~ms}^{-1}$. After equilibration we heat only the atoms located in the boundary boxes at the inlet and the outlet. As described in [7, atoms at the non-periodic boundary of the computational domain bounce with hard walls, which move with the local fluid velocity. At the end of each time step these walls are reset to their initial positions to maintain a fixed frame of reference. As a consequence some particles may remain outside the computational domain and are reinserted in regions of inflow using the Usher algorithm [14. This removal and insertion of particles is not symmetric and therefore we control the forces at the inlet and outlet of the computational domain separately with a $K_{P}$ of $0.0166 \frac{\mathrm{nm}^{3} \mathrm{~mol}}{\mathrm{amu} \mathrm{kJ}}$. We update the control force every $60 \mathrm{ps}$. In Fig.5 we show the external boundary force in the controlled and uncontrolled cases and the resulting reduced density and velocity profiles. The perturbed density in the uncontrolled case also leads to oscillations in the stream velocity $(u)$ of the molecules. The controller successfully eliminates the deviations from the target value for both quantities at both the inlet and outlet boundaries.

\section{Conclusions and Future Work}

We have presented a control algorithm to eliminate density fluctuations in the coupling of atomistic models with continuum descriptions of liquid water. A dynamic controller, based on the errors measured in the local fluid density, provides an appropriate boundary forcing, which applies the correct virial pressure to the system. The algorithm is validated for water at rest and it is shown to eliminate density oscillations with amplitude of the order of $10 \%$. In simulations of a uniform parallel flow the controller prevents the propagation of the perturbations, removing density and velocity oscillations of $11 \%$.

Future work will involve the extension of the method to non-equilibrium configurations and the treatment of long range electrostatics by the reaction field method [15] for multiscale modeling of ionic solvents.

\section{References}

1. O'Connell, S.T., Thompson, P.A.: Molecular Dynamics-Continuum Hybrid Computations: A Tool for Studying Complex Fluid Flow. Phys. Rev. E 52, 5792-5795 (1995)

2. Flekkoy, E.G., Wagner, G., Feder, J.: Hybrid Model for Combined Particle and Continuum Dynamics. Europhys. Lett. 52, 271-276 (2000)

3. Flekkoy, E.G., Delgado-Buscalioni, R., Coveney, P.V.: Flux Boundary Conditions in Particle Simulations. Phys. Rev. E. 72, 26703 (2000)

4. De Fabritiis, G., Delgado-Buscalioni, R., Coveney, P.V.: Multiscale Modeling of Liquids With Molecular Specificity. Phys. Rev. Lett. 97, 134501 (2006) 
5. Hadjiconstantinou, N.G.: Hybrid Atomistic-Continuum Formulations and the Moving Contact-Line Problem. J. Comput. Phys. 154, 245-265 (1999)

6. Nie, X.B., Chen, S.Y., Robbins, W.N.E., Robbins, M.O.: A Continuum and Molecular Dynamics Hybrid Method for Micro- and Nano-fluid Flow. J. Fluid Mech. 55, 55-64 (2004)

7. Werder, T., Walther, J.H., Koumoutsakos, P.: Hybrid Atomistic-Continuum Method for the Simulation of Dens Fluid Flows. J. Comput. Phys. 205, 373-390 (2005)

8. Kotsalis, E.M., Walther, J.H., Koumoutsakos, P.: Control of Density Fluctuations in Atomistic-Continuum Simulations of Dense Liquids. Phys. Rev. E 76, 16709 (2007)

9. Praprotnik, M., Matysiak, S.L., Delle Site, L., Kremer, K., Clementi, C.: Adaptive Resolution Simulation of Liquid Water. J. Phys. Condens. Matter 19, 292201 (2007)

10. Berendsen, H.J.C., Grigera, J.R., Straatsma, T.P.: The Missing Term in Effective Pair Potentials. J. Phys. Chem. 91, 6269 (1987)

11. Ryckaert, J.P., Cicotti, G., Berendsen, H.J.C.: Numerical Integration of the Cartesian Equations of Motion of a System with Constraints: Molecular Dynamics of n-alkanes. J. Comput. Phys. 23, 327-341 (1977)

12. Berendsen, H.J.C., Postma, J.P.M.: van Gunsteren,: The Missing Term in Effective Pair Potentials. J. Phys. Chem. 91, 6269 (1987)

13. Walther, J.H., Werder, T., Jaffe, R.L., Koumoutsakos, P.: Hydrodynamic Properties of Carbon Nanotubes. Phys. Rev. E 69, 62201 (2004)

14. De Fabritiis, G., Delgado-Buscalioni, R., Coveney, P.V.: Energy Controlled Insertion of Polar Molecules in Dense Fluids. J. Chem. Phys. 121, 12139 (2004)

15. Tironi, I.G., Sperb, R., Smith, P.E., van Gunsteren, W.F.: A Generalized Reaction Field Method for Molecular-Dynamics Simulations. J. Chem. Phys. 102, 5451-5459 (1995) 